\title{
Rationale and design of reGional network for Acute Myocardial Infarction (GAMI): protocol for a Prospective Cluster-randomized Intervention study of STEMI management
}

Yibo $\mathrm{He}$

Guangdong Provincial People's Hospital

Jin Liu

Guangdong Provincial People's Hospital

Liwei Liu

Guangdong Provincial People's Hospital

Feier Song

Guangdong Provincial People's Hospital

Ming Ying

Guangdong Provincial People's Hospital

Guoli Sun

Guangdong Provincial People's Hospital

Yihang Ling

Guangdong Provincial People's Hospital

Yan Liang

Maoming People's Hospital

Jianfeng Ye

Dongguan Hospital of Traditional Chinese Medicine

Yunzhao Hu

Shunde Hospital of Southern Medical University

Mingcai Song

Guangzhou Panyu Central Hospital

Qingbo Xu

Maoming People's Hospital

Cong Chen

Maoming People's Hospital

Guoqin Chen

Guangzhou Panyu Central Hospital

Ning Tan

Guangdong Provincial People's Hospital 


\section{Jiyan Chen}

Guangdong Provincial People's Hospital

\section{Yong Liu}

Guangdong Provincial People's Hospital

Shiqun Chen ( $\nabla$ shiqunchen@126.com )

Guangdong Provincial People's Hospital

\section{Research Article}

Keywords: acute myocardial infarction, ST-segment Elevation Myocardial Infarction, regional intervention, regional network construction, cohort study

Posted Date: November 11th, 2021

DOl: https://doi.org/10.21203/rs.3.rs-682606/v1

License: (c) (1) This work is licensed under a Creative Commons Attribution 4.0 International License.

Read Full License 


\section{Abstract}

\section{Background}

Timely revascularization is essential for ST-segment elevation myocardial infarction (STEMI) patients to reduced mortality rate. However, it's seldom achieved due to patient and medical system-level barriers in China, especially in the rural area. It's needed to establish a regionally coordinated network to promote the early revascularization rate for STEMI patients.

\section{Methods}

Regional network for Acute Myocardial Infarction (GAMI) is a multi-center, prospective, intervention study with regional intervention. It was carried out in 8 centers by 1:1 cluster randomization in south China with diverse economic and medical levels since 2016. The regional intervention were carried out in the intervention centers, which consisted of intensive public education and regional network construction for STEMI treatment, including community and online propaganda to raised patients' awareness of the treatment for STEMI and establishment of regional clinic-EMS-PCI transfer network for timely primary revascularization. Consecutive acute STEMI patients were enrolled for assessment of regional intervention according to the key performance indicators. The primary study endpoint was reperfusion rate, and secondary endpoints included change of pre-hospital delay, changes of in-hospital delay time and in-hospital outcomes. All patients were followed up for more than 1 year.

Discussion

The GAMI study is a prospective cluster-randomized intervention study, which would provide helpful evaluation of the effectiveness of enhanced public education and regional network construction on regional STEMI treatment

Trial registration: Clinical Trials, NCT03928119. Registered , 25 April, 2019ه

https://clinicaltrials.gov/ct2/show/NCT03928119?id=NCT03928119\&draw=2\&rank=1

\section{Article Summary}

\section{Strengths and limitations of this study:}

1. This study is the first attempt to implement regional strategies on patients and system-level barriers to improving STEMI treatment delays in China.

2. The study adopts an annual assessment to evaluate the effects of regional strategies for further improvement.

3. This study included both rural and urban areas to explore comprehensive strategies in line with the actual situation of STEMI treatment in China. 
4.Further studies are needed to confirm the effectiveness of the regional strategy of STEMI treatment carried out in this study.

\section{Introduction}

The incidence and mortality of acute myocardial infarction (AMI) in China have increased year by year. The annual report of China cardiovascular disease indicated that approximately 890,000 patients died of AMI in 2016[1]. Since 2012, the mortality rate of acute myocardial infarction in rural areas had exceeded that in urban areas and presented ascending trend by the following years. Measures to reduce the mortality of acute myocardial infarction were in urgent need.

Early reperfusion therapy is essential for AMI treatment, especially for ST-segment elevation myocardial infarction (STEMI) patients, which accounted for more than $80 \%$ of AMI in China and maintained increasing[2]. Although the number of percutaneous coronary intervention (PCI) continued to increase, primary $\mathrm{PCl}$ has not increased significantly in the past decade, the overall STEMI in-hospital mortality rate has not changed[3][4]. The first Chest Pain Center (CPC) in China which aimed to shorten the diagnosis and treatment delay was set up in 2011 and promote to the whole country gradually. The risks of major adverse cardiovascular events (MACEs) and all-cause death were gradually decreased as percutaneous coronary intervention (PCI) capable hospitals achieving CPC accreditation[5]. However, there was still not enough CPC accredited hospital with sufficient medical capability to provide with efficient treatment for STEMI patients in most areas, particularly in the rural and remote areas. It has been reported that STEMI patients averagely took 170 minutes to go to the hospital for the first medical contact, and only $28.2 \%$ of STEMI accepted PCI treatment within the goal time recommended by international guidelines[6][7]. In addition, our former qualitative study[8] had suggested that actions were needed to overcome patients and system-level barriers, mainly including enhanced public education and regional capacity for STEMI treatment. More effort should be made to elevate the efficacy of STEMI treatment in an extensive region including rural and remote areas. However, it's not clear how systematic enhanced public education and regional network construction for AMI treatment would affect the treatment quality of STEMI patients in China.

Herein, we performed the GAMI project to construct a comprehensive regional network comprising enhanced public education and regional network construction for STEMI treatment to overcome the patient-level and system-level barriers.

\section{Methods}

\section{Study design}

The GAMI project is a multi-center, prospective, cluster-randomized intervention study with regional intervention conducted in 8 regions which represented different economic and medical resource levels in south China since 2016. The objective of the study was to evaluate the effectiveness of regional 
interventions, including enhanced public education and regional network construction, on the change of treatment delay of the STEMI patient's treatment. Consecutive STEMI patients were enrolled from the major $\mathrm{PCl}$ centers of the regions. By analyzing the key performance indicators such as reperfusion rate within 12 hours since symptom onset, change of pre-hospital delay, changes of in-hospital delay time and in-hospital outcomes between the intervention groups and control groups, the effectiveness of the intervention measures was evaluated for further optimization. (see study design overview in Fig. 1)

\section{Study regions and randomization}

The study was carried out in 8 regions which represented various socioeconomic conditions as well as medical resource level in China. The regions were selected based on geographic location, urbanization, medical capacity to support timely treatment of STEMI. (Fig. 2) In addition, all these cities were willing to participate in the study to improve the treatment condition for STEMI patients. Stratified cluster randomization was performed according to the composite evaluation of the socioeconomic conditions, urbanization and medical capacity of the local regions. Guangzhou, Panyu, Shenzhen and Foshan regions were considered as developed area of medical capacity of STEMI treatment. Among them, Panyu and Foshan were randomized to the intervention groups while Guangzhou and Shenzhen were in the control group. Dongguan and Yangjiang were considered as the developing areas, Dongguan were randomized to the intervention group and Yangjiang were randomized to the controlled group. Maoming and Longyan were considered underdeveloped regions of medical capacity for STEMI treatment, of which Maoming were randomized to the intervention group and Longyan were randomized to the controlled group.

\section{Patient enrollment}

The major $\mathrm{PCl}$ centers of the study regions were included in the study to be in charge of local interventions of enhanced public education and regional network construction. (Listed in supplementary

1) Consecutive STEMI patients admitted to the $\mathrm{PCl}$ centers through the emergency department were included to evaluate the change of treatment delay. The diagnosis of STEMI must met the third Universal Definition for Myocardial Infarction. Relevant medical information were recorded and collected by the reception doctors, including time of symptom onset, time of arrival at the hospital, time of balloon dilatation. Patients were excluded if the timings of the treatment were missing. The treatment process is completely decided by the doctors in charge according to the patient's condition, without interference by the study enrollment.

\section{Regional intervention group}

Enhanced public education

Enhanced public health education for acute myocardial infarction was carried out in a variety of ways, including theme lectures in communities, brochures distribution, messaging education, push service of mobile applications, television and newspaper advertisement and so on. Cooperated with the local 
community medical service center, targeted education on the basic knowledge, self-identification, and key points for timely treatment of acute myocardial infarction were conducted. The frequency of education was determined according to the actual situation of each region, the content and attendance number were recorded after each education.

Regional network construction

The regional network construction included actions as follow: 1) training in the diagnosis and treatment of STEMI patients in primary and secondary hospitals, developing a unified and effective process of first medical contact; 2) construct the efficient "green" first-aid channel for STEMI treatment in PCI hospitals, shortening the delay time of door-to-balloon; 3) cooperated with the local 120 emergency center to establish a co-working network, which comprised of all the three-level hospitals in the region, and standardized route as well as procedure for prompt STMEI patients transfer. The practical implementation plan was carried out according to the actual situation of each region. For example, in order to improve the rate of early reperfusion therapy in regions where transfer of STEMI patients from first contact primary hospital to tertiary $\mathrm{PCl}$ hospital was too remote to complete within 2 hours, several primary hospitals will be selected to construct as a thrombolytic treatment unit for transitional reperfusion therapy.

\section{Study outcomes and follow-up management}

The study outcomes are as follows: the primary outcome was defined as reperfusion rate, defined as the proportion ratio of timely reperfusion with 12 hour since symptoms onset to revascularization among the whole enrolled population; secondary outcomes were defined as (1) change of pre-hospital delay of symptom onset to PCl hospital-arrival time; (2) Changes of in-hospital delay time, including door-toballoon time; (3) in-hospital outcomes, including MACE events (consist of in-hospital death, stoke and recurrence of myocardial infarction), in-hospital death, and treatment withdrawal.. Telephone interviews or clinical interviews were conducted for all participated STEMI patients for at least 1 year (at 3, 6, 12 months since enrollment within the first year), and annual follow-ups were performed after that.

\section{Data collection}

Trained physicians or study nurses collected data with a data collection card during patients' hospitalization by medical record review. Items of interest are 1) demography data, 2) preexisting risk factors and comorbidities, 3) medical treatments and interventions during hospitalization, 5) clinical complications, 6) medication before and during hospitalization and at hospital discharge, 7) symptomonset to the first medical contact time, door-in-door-out time, door-to-balloon time, 8) laboratory information. The key timing data of STEMI onset and treatment are precisely recorded.

The timing of treatment, such as admission time, first ECG time, wire-crossing time of PCl treatment, were recorded in a STEMI patients data collection card by a medical staff alongside since the STEMI patients were admitted to the emergency department (Fig. 3). If the patient was transferred by the ambulance from emergency medical service, the patient and the paramedic on the ambulance would be asked for the 
timing record before the admission, such as the time to call the ambulance. The characteristic information of the patient's hospitalization is collected through the records of the hospital information system and entered into the electronic data capture system. The STEMI patients were followed up through outpatient service or telephone calls at 1 month, 3 months, 6 months and 1 year, and the followup medication and adverse events were collected and entered into the electronic data capture system

\section{Data management}

Critical timing data of the STEMI treatment course were recorded on the STEMI timing data collection card. Once patients with chest pain were diagnosed with STEMI in the emergency department, clinical doctors started to record the information on the STEMI treatment timing according to the patients' inquiry. The timings of STEMI treatment were recorded by the emergency doctors, the cardiologists, and the catheter surgeons or nurses respectively. The timing data collection cards were finally collected by the research assistants and the information integrity would be checked. If there was any information missing, the patient or relevant physician would be promptly asked to fill up. The assistant filled in the complete STEMI information card with the CRF and double-check to ensure that the information is accurate, and the STEMI information registration card is reserved for verification afterward.

Inpatient data of STEMI patients were recorded in the Hospital Information System (HIS) according to the routine medical treatment process. After discharge, all the STEMI patients' case files were scanned and stored in the archive. Two research assistants recorded the in-hospital information of STEMI patients on the Clinical Research Form (CRF) based on the record of HIS files separately, checked the inconsistent data to verify the accuracy. When abnormal data was found, research doctors were promptly informed to retrieve the original data and make the correction. All the data recorded in the CRF were then entered on the online Electronic Data Capture (EDC) system. When the entry was completed, the data administrator would perform the database inspection and reported the abnormal data for inquiry, which consisted of content completion check, outlier data check, logical consistency check, etc. All the data with inquiry were inspected again to make sure veracity.

We established different methods for quality assurance. 1) Standard Operating Procedures (SOP) To reduce variability in data collection, all reporting institutions (hospitals, health departments) fill in all questionnaires according to standard operating procedures. 2) Data-Completeness All records are regularly checked for errors and inconsistencies. 3) Case-Reporting-Completeness To check the completeness of case reporting, we compared the number of all patients who were annually discharged from all the collaborating hospitals with a diagnosis of STEMI with the number of registered cases.

Data safety and confidentiality

All the data records, including the STEMI timing record card and the clinical research form, are encrypted and stored in the electronic data capture system, all medical staff members had to use their unique passwords to log into the online EDC system for data access. The paper data records were archived and stored in a special archive. 
Sample size of 4000 in the intervention group and 4000 in the control group, which were obtained by sampling 4 clusters with 1000 subjects each in the intervention group and 4 clusters with 1000 subjects each in the control group, achieve $83.475 \%$ power to detect a superiority margin difference between the group proportions of 0 . The proportion in the intervention group is assumed to be 0.34 under the null hypothesis and 0.5 under the alternative hypothesis. The proportion in the control group is 0.34 . The test statistic used is the one-sided Z-Test (Pooled). The intra-cluster correlation is 0.03 , and the significance level of the test is 0.05

\section{Statistical analysis}

Continuous variables are represented in form of mean \pm standard deviation or median and quartile according to whether they conform to a normal distribution, categorical variables are expressed in percentiles.Trends in baseline characteristics are tested using the Cochran-Armitage test for categorical variables and the Mann-Kendall test for continuous variables. To test for the in-hospital outcomes, the first year was considered as the reference, we constructed four indicator variables representing the following years, the time interval for each variable is 1 year. We performed logistic regression to test for in-hospital outcome, including indicators for time as key explanatory variables after adjusting for patients' demographics, medical history, and clinical characteristics at admission. Odds ratios (ORs) and $95 \% \mathrm{Cl}$ from the multivariate logistic analysis related to the year indicators will be reported as well.

Subgroup analysis of age, sex and different regions will be performed for additional exploratory analyses. All tests were two-tailed, p-values less than 0.05 are considered statistically significant. The statistical analyses would be performed using R software (version 3.1.2; R Foundation for Statistical Computing, Vienna, Austria).

\section{Dissemination}

Findings will be disseminated in future peer-reviewed papers and will inform strategies aiming at developing a collaborative regional network for STEMI management and education to reduce the delay time and STEMI mortality.

\section{Patient and Public Involvement}

The study involved no patient or public on the development of the research question, outcome measures, study design, recruitment, conduct and result dissemination.

\section{Discussion}

GAMI project is a prospective, multicenter cluster-randomized intervention study with regional tentative strategies to reduce system-level and patient-level delay so that to establish an effective regional network for timely reperfusion treatment of STEMI patients in China. It was implemented in 8 regions which represented different economy level and urbanization in southern China, which would hopefully provide with useful experience for regional strategy formulation of STEMI treatment and promote to the whole 
nation. Through this study, we will be able to discover the impact of regional STEMI treatment interventions on the treatment effect and prognosis of STEMI patients, which will provide a reference for the public health construction of STEMI treatment. Meanwhile, it's able to investigate factors that influence morbidity and mortality rates due to STEMI under different economic levels and medical conditions.

Cardiovascular disease (CVD) remains a predominant global burden of disease, and it is the largest single contributor to global mortality[9]. As for China, CVD is also the main burden of disease, leading to more than $40 \%$ of deaths in China[10][11]. According to previous statistics, there are two hundred million CVD patients in China, and the China-PEACE study [12]reported that the admission rate for ST-segment elevation myocardial infarction grew rapidly from 2001 to 2011. Despite more developed medical treatments emerged, acute myocardial infarction remains the major cause of re-hospitalization, heart failure, and death[13]. Numerous studies aimed to lessen the incidence of adverse outcome of AMI and found out that myocardial reperfusion delay in ST-segment elevation myocardial infarction (STEMI) acted as one of the most important factors[14], and the interval between symptom onset and reperfusion therapy is recognized as a determinant of prognosis in STEMI patients[15]. The reasons for reperfusion delay include Patient Delay Time (PDT- symptom onset to first medical contact) and System Delay Time (SDT - from first medical contact to arterial reperfusion)[16]. The awareness of the high risk of AMI played a significant role in patient delay, for example, attributing the symptoms to other conditions, fear of disturbing others, judge that the symptoms are a false alarm, lack of knowledge of the importance of quick action and so on[17]. As for system delay, pre-hospital transfer delay accounts for a major problem, for example, patients living in rural areas were diagnosed with AMI and usually experienced longer retention at local healthcare institutes rather than quick transfer to $\mathrm{PCl}$ capable hospital[18][19]. Therefore, except for advanced treatments, setting up an effective regional network reducing PDT and SDT to decrease reperfusion delay is highly necessary.

Regarding the treatment of acute myocardial infarction, the CAMI study and China-peace project, which were both observational studies, reveal the conditions and factors of AMI treatment in China. Untypical chest pain, unaware of cardiac symptoms and prolong delay due to rural remote distance and so on were reported affecting timely AMI treatment[20][21]. The construction of Chest Pain Center in China emphasizes accredited criteria of in-hospital treatment procedures such as door-to-balloon time, which had been proven to reduce the mortality rate of STEMI patients admitted to accredited hospitals[5]. However, there is no systematic and standardized regional acute myocardial infarction treatment strategy project yet to deal with problems in patient delays as well as treatment and transfer in the rural and remote primary health clinic. Timothy $D$. Henry et al designed a standardized protocol and integrated system of transfer for patients with STEMI to be proven effective, where the simplify the initial evaluation, treatment and transfer in the community hospital were carried out in Minneapolis, USA[22]. In Sweden, the health care quality of STEMI was popularly followed, making newspaper, TV and radio headlines to attract public attention and promote the education of AMI treatment[23]. Similar measures should be carried out in China, and relevant studies are needed to verify the effectiveness of these measures. 
So far, there is no research focus on the construction of regional network to deal with both system-level and patient-level delays of STEMI treatment in China. GAMI study is the first project attempting to establish regional network comprising both extensive public education and medical training and transfer collaboration between primary health clinic and $\mathrm{PCI}$ capable hospital to reduce reperfusion delay so that to improve the clinical outcome of STEMI treatment. The extensive public education we adopted included TV promotion, internet push, community lectures, and advertising posters to attract the public's attention to the treatment of acute myocardial infarction. Various forms of publicity have proven to be beneficial to public health awareness[24]. In particular, our measures have been further extended to rural areas, so that people in rural and remote areas can have access to health care information. STEMI medical training were carried out for the physician in primary health clinic would elevate their ability to diagnose and treat STEMI which would also reduce the system-level delay[25]. The collaboration transfer network we set between primary health clinic and PCl capable hospital would help transfer STEMI patients more quickly to reach revascularization treatment, reducing retention delay in the primary health clinic where might even lack of the fundamental thrombolytic therapy.

Moreover, this project is an ongoing project with STEMI patient enrollment and regional intervention. We would evaluate the effectiveness of the acute myocardial infarction treatment network in these 4 cities with different economic levels and medical resource capabilities through comparing the key performance indicators annually, and further optimize the development of the network construction. In these ways, we might establish an effective regional network for STEMI treatment in line with the regional economic level and medical source capability, and hopefully promote other cities in China.

However, there are a few limitations in this study. Firstly, measures in the regional network construct are not specialized for individual STEMI patients, and cohort studies need to be further carried out to clarify the effectiveness of the measures for the public and primary health clinic. Secondly, the STEMI patients enrolled in the $\mathrm{PCl}$ hospital may not be able to reflect the situation of all STEMI patients in the region. Also, measures of this project might take several years to observe changes in the overall pattern of treatment of STEMI. Nonetheless, the GAMI study is the first attempt to establish a regional network to solve the patient and system-level barrier of treatment delay of STEMI patients, it can provide useful experience in setting up a regional STEMI treatment system that meets the actual situation under conditions of various economic levels and medical source capability.

\section{Conclusion}

GAMI is a prospective cluster-randomized intervention study with regional intervention comprising enhanced public education, regional network construction for STEMI patients, with a goal to establish a novel regional strategy for the treatment of acute myocardial infarction with less delay and mortality.

\section{Trial Status}


The protocol reported here is version 1.0, dated December 2015. Trial enrollment began on June 2016 and finished on June 2021. The follow-up visit is anticipated to finished on June 2022.

\section{Declarations}

\section{Ethics approval}

GAMI was approved by the central ethics committee at the Guangdong Provincial People's Hospital with an informed consent exemption (No. GDREC2015433H). All data in the study will be collected following approval by the corresponding ethics committees of the participating centers. The information of enrolled STEMI patients about STEMI management was collected confidentially and stored in an encrypted database. An independent data monitoring committee will be responsible for the review of the ongoing safety of patients enrolled in the study.

\section{Consent for publication}

Not applicable.

\section{Competing interests}

The authors declare that they have no competing interests.

\section{Funding}

This study was supported by grants from the Science and Technology Planning Project of Guangdong Province (No.2014B070706010); Multi-center study on key techniques for prevention, diagnosis and treatment of high risk coronary artery disease(DFJH2020026); Study on the function and mechanism of the potential target for early warning of cardiorenal syndrome after acute myocardial infarction based on transmoomics(DFJH201919); Natural Science Foundation of Guangdong Province General Project(2020A1515010940); Guangdong Provincial Science and Technology Plan Project (2017B030314041)

\section{Authors' contributions}

$\mathrm{YL}$ and $\mathrm{JC}$ had the original idea.

$\mathrm{YH}, \mathrm{JL}, \mathrm{NT}$ contributed to the study design.

$\mathrm{SC}, \mathrm{YH}, \mathrm{JL}$ were involved in the design of the statistical analysis approach.

$\mathrm{LL}, \mathrm{FS}, \mathrm{MY}, \mathrm{GS}, \mathrm{Y}-\mathrm{hL}$ were involved in the literature review and developing study instruments and materials.

$Y L, J Y, Y H, M S, Q X, C C$ are site PI who participated in conducting the trial and acquisition of data. 
YH, LL, FS, MY, Y-hL drafted and revised the manuscript. All authors contributed critical intellectual input and approved the final manuscript.

\section{Acknowledgments}

Not applicable.

\section{References}

1. National Center for Cardiovascular Disease., REPORT ON CARDIOVASCULAR DISEASES IN CHINA(2018). Encyclopedia of China Publishing House, 2019.

2. Gao R, et al. Prospective observational study of acute coronary syndromes in China: practice patterns and outcomes. Heart. 2008;94(5):554-60.

3. Li J, et al. ST-segment elevation myocardial infarction in China from 2001 to 2011 (the China PEACERetrospective Acute Myocardial Infarction Study): a retrospective analysis of hospital data. Lancet. 2015;385(9966):441-51.

4. Zheng $X$, et al. Coronary Catheterization and Percutaneous Coronary Intervention in China: 10-Year Results From the China PEACE-Retrospective CathPCI Study. JAMA Intern Med. 2016;176(4):512-21.

5. Fan F, et al. Chest Pain Center Accreditation Is Associated With Improved In-Hospital Outcomes of Acute Myocardial Infarction Patients in China: Findings From the CCC-ACS Project. J Am Heart Assoc. 2019;8(21):e013384.

6. Zhang Y, et al., Treatment Delay and Reperfusion Management of Acute ST-segment Elevation Myocardial Infarction - analysis of the China STEMI Care Project Phase 1 (CSCAP-1). Qjm, 2020.

7. Ibanez B, et al. 2017 ESC Guidelines for the management of acute myocardial infarction in patients presenting with ST-segment elevation: The Task Force for the management of acute myocardial infarction in patients presenting with ST-segment elevation of the European Society of Cardiology (ESC). Eur Heart J. 2018;39(2):119-77.

8. Yin $X$, et al. Patient-level and system-level barriers associated with treatment delays for ST elevation myocardial infarction in China. Heart. 2020;106(19):1477-82.

9. Sacco RL, et al. The Heart of 25 by 25: Achieving the Goal of Reducing Global and Regional Premature Deaths From Cardiovascular Diseases and Stroke: A Modeling Study From the American Heart Association and World Heart Federation. Circulation. 2016;133(23):e674-90.

10. Zhou M, et al. Cause-specific mortality for 240 causes in China during 1990-2013: a systematic subnational analysis for the Global Burden of Disease Study 2013. Lancet. 2016;387(10015):25172.

11. Du X, et al. Epidemiology of Cardiovascular Disease in China and Opportunities for Improvement: JACC International. J Am Coll Cardiol. 2019;73(24):3135-47.

12. Retraction. and republication-ST-segment elevation myocardial infarction in China from 2001 to 2011 (the China PEACE-Retrospective Acute Myocardial Infarction Study): a retrospective analysis of 
hospital data. Lancet. 2015;385(9966):402.

13. Dreyer RP, et al. Myocardial infarction with non-obstructive coronary arteries as compared with myocardial infarction and obstructive coronary disease: outcomes in a Medicare population. European heart journal. 2020;41(7):870-8.

14. Schoos MM, et al. Reperfusion delay in patients treated with primary percutaneous coronary intervention: insight from a real world Danish ST-segment elevation myocardial infarction population in the era of telemedicine. European heart journal. Acute cardiovascular care. 2012;1(3):200-9.

15. Gersh BJ, Stone GW. Pharmacological facilitation of coronary intervention in ST-segment elevation myocardial infarction: time is of the essence. JACC Cardiovasc Intervent. 2010;3(12):1292-4.

16. Rathore SS, et al., Association of door-to-balloon time and mortality in patients admitted to hospital with ST elevation myocardial infarction: national cohort study. BMJ (Clinical research ed.), 2009. 338: p. b1807.

17. Vavouranakis I, et al. Delays incurred during acute myocardial infarction: a comparative study of rural and urban populations in Greece. Rural Remote Health. 2010;10(2):1271.

18. Sørensen JT, et al. Urban and rural implementation of pre-hospital diagnosis and direct referral for primary percutaneous coronary intervention in patients with acute ST-elevation myocardial infarction. European heart journal. 2011;32(4):430-6.

19. Fu R, et al., Differences in symptoms and pre-hospital delay among acute myocardial infarction patients according to ST-segment elevation on electrocardiogram: an analysis of China Acute Myocardial Infarction (CAMI) registry. Chinese medical journal, 2019. 132(5): p. 519-524.

20. Guan W, et al. Time to hospital arrival among patients with acute myocardial infarction in China: a report from China PEACE prospective study. European heart journal. Quality of care clinical outcomes. 2019;5(1):63-71.

21. Henry TD, et al. Design of a standardized system for transfer of patients with ST-elevation myocardial infarction for percutaneous coronary intervention. Am Heart J. 2005;150(3):373-84.

22. Knot J, et al. How to set up an effective national primary angioplasty network: lessons learned from five European countries. Eurolntervention. 2009;5(3):p. 299, 301-9.

23. Xu Q, Huang Y, Chen B. Comprehensive assessment of health education and health promotion in five non-communicable disease demonstration districts in China: a cross-sectional study. BMJ open. 2017;7(12):e015943.

24. Masuda J, et al. Rural-Urban Disparity in Emergency Care for Acute Myocardial Infarction in Japan. Circ J. 2018;82(6):1666-74.

\section{Figures}




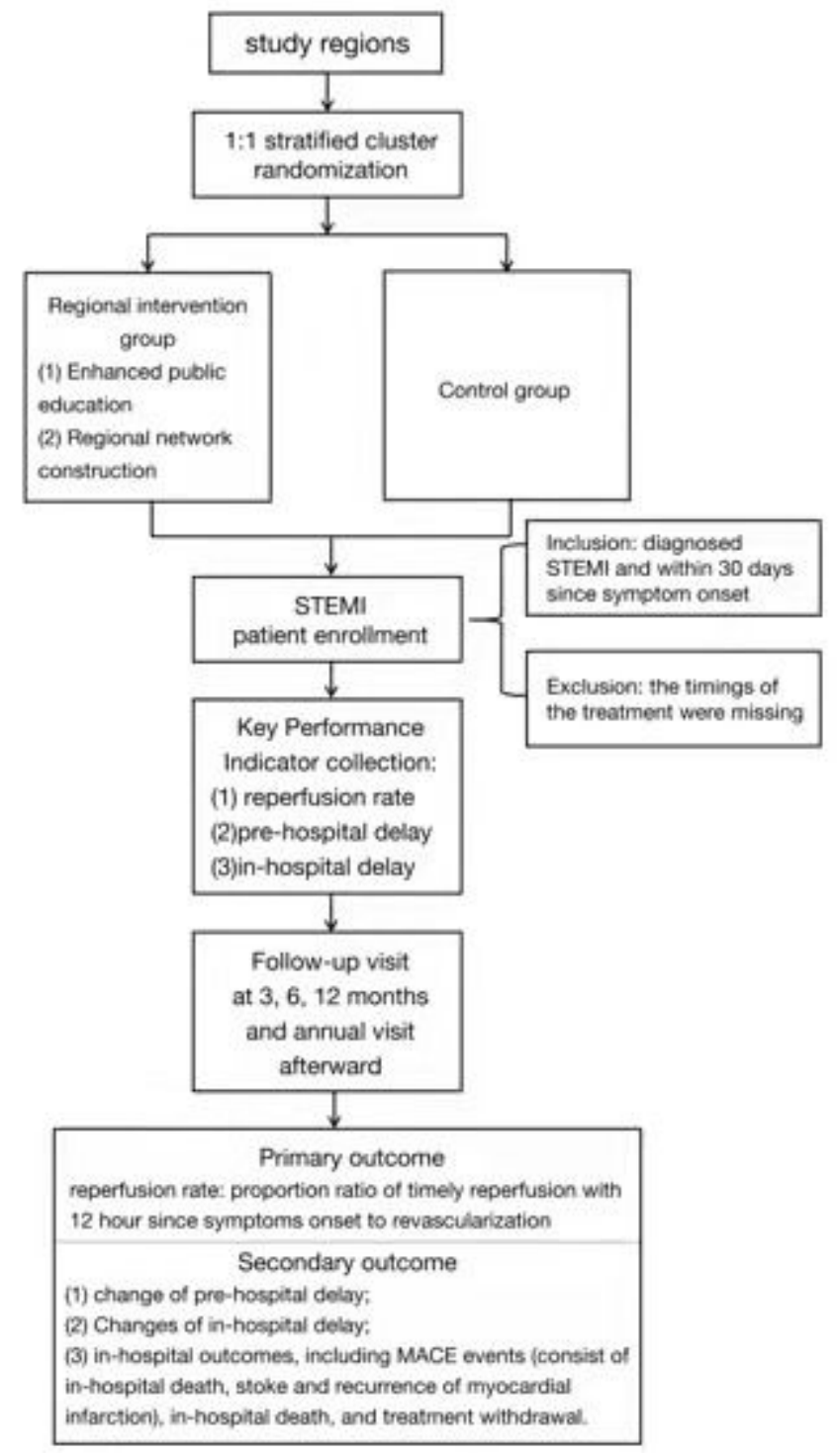

Figure 1

flow chart of GAMI study 


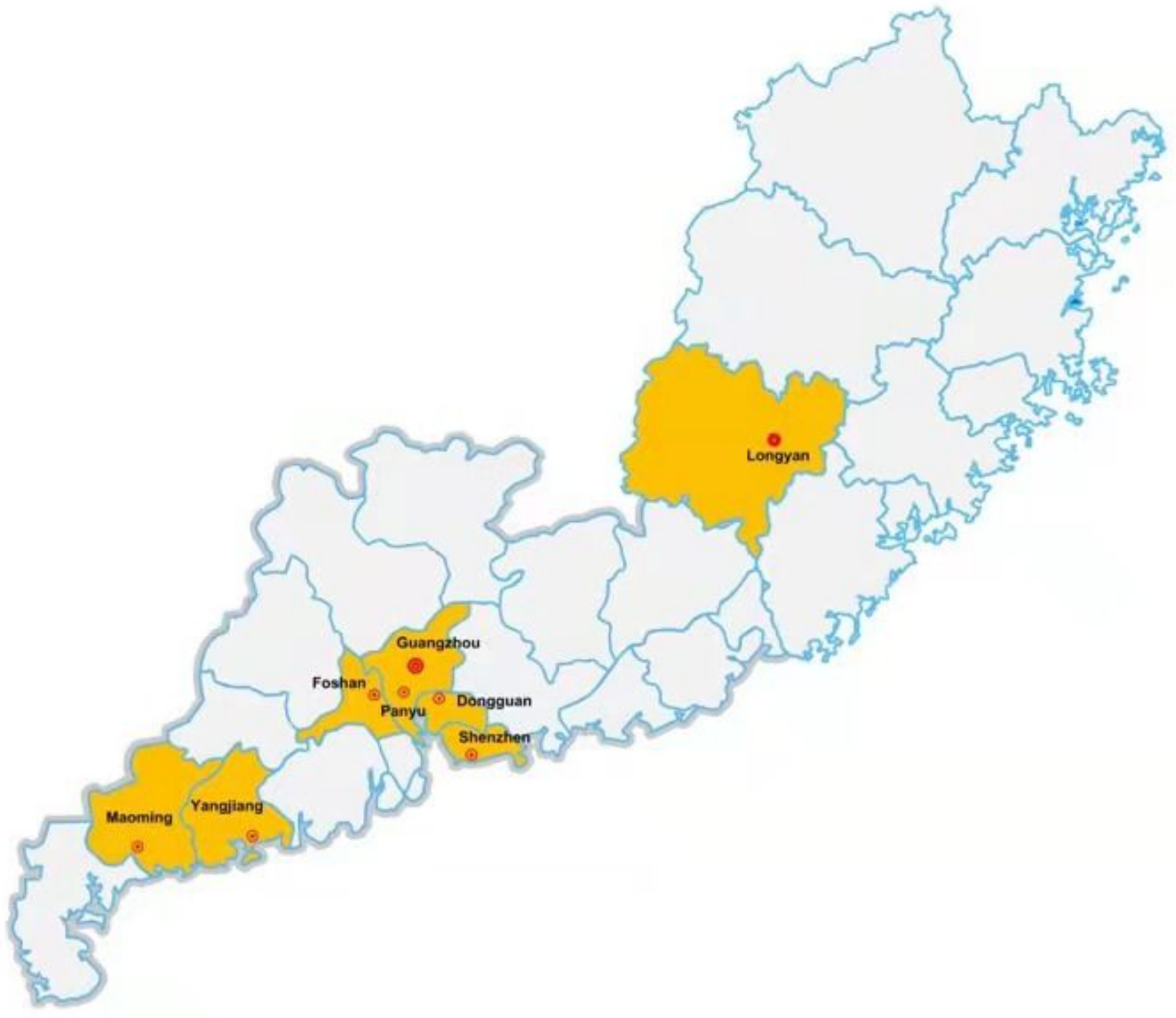

Figure 2

Geographic distribution of study regions. 
STEMI Data Collection Card

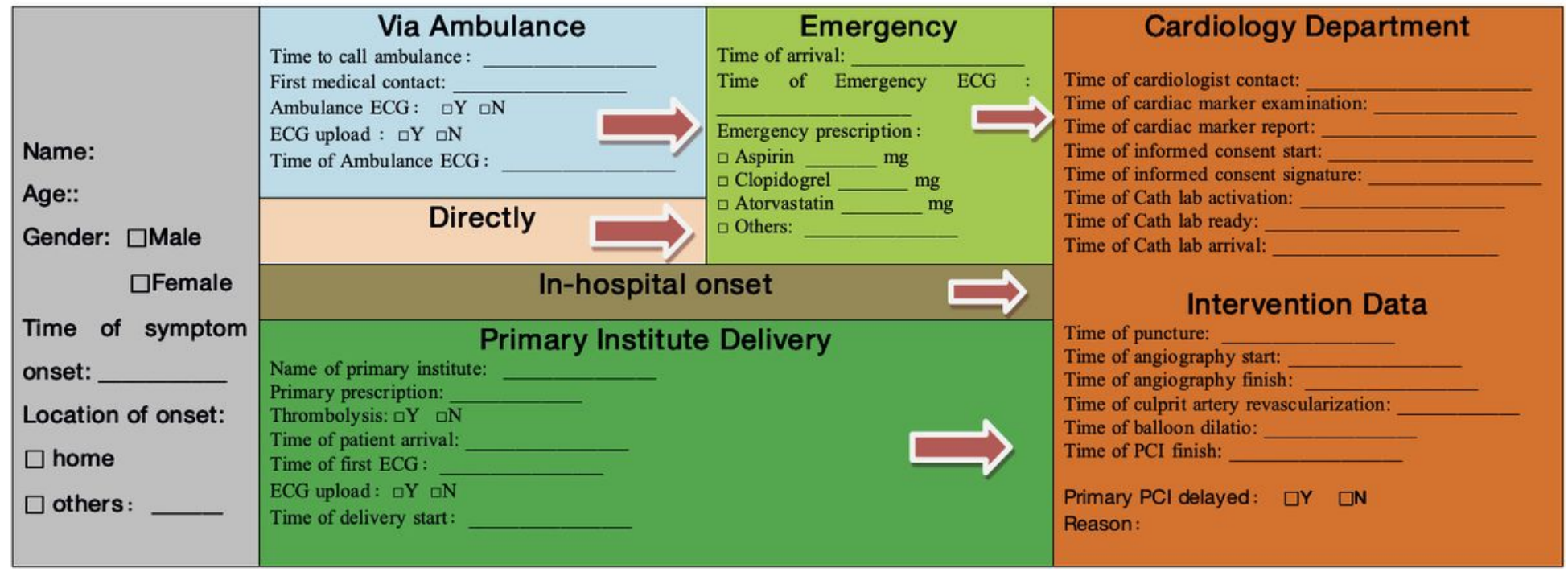

\section{Figure 3}

STEMI patients data collection card

\section{Supplementary Files}

This is a list of supplementary files associated with this preprint. Click to download.

- SupplementaryMaterial.docx 